\title{
Overcoming the stratum corneum barrier: a nano approach
}

\author{
Rinti Banerjee
}

Published online: 17 April 2013

(C) Controlled Release Society 2013

\section{A brick and mortar wall}

The skin represents a large surface area which is easily accessible and can potentially serve as a very attractive non-invasive route of delivery of drugs. However, there have been very few technologies exploiting this route of drug delivery commercially. One of the main reasons for the limited success is the presence of the "brick and mortar" barrier of the stratum corneum which acts as a defensive wall that needs to be overcome in order to improve the efficiency of transdermal drug delivery. Understanding the organisation of the stratum corneum can help in designing more efficient drug carriers which are based on their biophysical effects on the stratum corneum barrier.

\section{Short and long of the stratum corneum}

The stratum corneum consists of dead cells or corneocytes which are embedded in a special lipid rich matrix. The composition of the lipids in the stratum corneum consists mainly of ceramides, cholesterol and saturated long chain fatty acids. The lamellar organisation of the stratum corneum consists of the short periodicity phase with repeat units at 6-nm distances and a long periodicity phase with repeat distances of $13 \mathrm{~nm}$ [1]. In addition to the lamellar organisation, there is also a very close lateral packing associated with the lipid molecules of the stratum corneum. An orthorhombic lateral packing is associated predominantly with the human stratum corneum and plays a very important role as a barrier to the transport of moieties across the skin.

R. Banerjee $(\bowtie)$

Department of Biosciences and Bioengineering, Indian Institute of Technology Bombay, Mumbai 400076, India

e-mail: rinti@iitb.ac.in
Nanotechnology strategies can be designed that modulate the packing and phases of the lipid component of the stratum corneum a process referred to as fluidisation. While attractive, the changes introduced must be reversible or short-lived so that the protective barrier function that allows the skin to ward off unwanted agents remains intact.

\section{Role of nanotechnology}

Nanoparticles have the potential to fluidise the stratum corneum lipid packing or to penetrate the skin along the hair follicles and sweat glands. Nanoparticles can be designed to penetrate through the stratum corneum for deeper penetration into the dermis and thereby gaining access to a rich capillary network to reach the systemic circulation. On the one hand, this technology has opened up a large number of exciting possibilities of exploiting the stratum corneum barrier more efficiently for drug delivery while on the other hand, the ability to penetrate the barrier accidentally may lead to controversies over toxicity concerns with non-degradable inorganic nanoparticles like titanium dioxide nanoparticles in sunscreens, particularly if surface modified with agents which may act as penetration enhancers [2].

\section{Optimal strategies}

Effect of nanoparticle properties

The success of nanotechnology for transdermal applications depends on defining and optimising the nanomaterial characteristics such that penetration through the skin can be enhanced while avoiding any toxicity concerns. The size, shape, charge and degree of unsaturation all play a 
role in determining the cellular effects of the nanoparticles. Unsaturated phospholipids and fatty acids play an important role in the fluidisation of the stratum corneum and hence are preferred components of lipid-based nanoparticles for transdermal delivery.

Liposomes are an important category of lipid nanoparticles for dermal penetration as they can exploit the fluidisation effects of the lipids. The flexible membrane of the liposome, its size and the unsaturation of the lipids help to alter the spacing of the stratum corneum lipid barrier and facilitate entry of drugs and actives through the skin. Apart from liposomes, other nanostructures of lipids having higher stability and ease of large-scale manufacturing like solid lipid nanoparticles, nanostructured lipid carriers and micellar nanoparticle technologies also show promise for dermal delivery. For example, both solid lipid nanoparticles and nanostructured lipid carriers of 100-250 nm using various oils and fatty acids were found to be suitable for dermal delivery of trans-retinoic acid [3].

\section{Combination of triggers}

Though nanoparticles can inherently penetrate through skin if suitably designed, certain physicochemical triggers can be used along with the nanoparticles to enhance their penetration. Combination of physicochemical triggers and nanoparticles has been exploited for synergistic effects on drug penetration. For example, negatively charged poly(lactic-co-glycolic acid) (PLGA) nanoparticles of $100 \mathrm{~nm}$ when combined with iontophoresis have been found to enhance the dermal permeation of indomethacin loaded within the nanoparticles several folds [4].

Recent studies have found that even rigid nanoparticles can pass through the barriers of the skin when accompanied by ultrasound triggers. Application of ultrasound and sodium lauryl sulphate as a pre-treatment was found to enhance the dermal penetration of quantum dots by 500 to $1,300 \%$ [5]. This was a major advancement in our technologies for dermal penetration and caused several folds increase in the permeation flux of the drugs through skin.

In another study, photothermal ablation of the stratum corneum using light activated nanoparticles has been used to enhance the penetration of drugs through the skin. However, hollow copper sulphide nanoparticles were used for photothermal activation and there are concerns regarding the toxicity and biodegradability of these nanoparticles [6].

In addition to physical triggers, certain penetration enhancing moieties may also be combined with nanoparticles for increased permeation. Cell penetrating peptides have been investigated as one such strategy and have been used to decorate the surface of nanoparticles to enhance their cellular penetration [7].

\section{Microneedle technology}

Microneedle-based platforms have also been exploited to directly deliver drugs and vaccines through the stratum corneum in the dermis. The technology comprises tiny patches of microneedle arrays made of biodegradable polymers which project painlessly through the skin and dissolve in a short period releasing vaccines, drugs or hormones embedded within them. The technology has been used to develop needle-free vaccines which do not require cold storage [8].

\section{Exploiting various routes through the skin}

Since the stratum corneum is the main barrier of skin penetration, most strategies involve optimisation of nanoparticles to enhance the fluidisation or penetration through the stratum corneum. In addition to these strategies, one can also design nanostructures to exploit the follicular and sebaceous routes of drug delivery. Solid polymeric nanoparticles accumulate at hair follicle openings and penetrate deeper into the follicle than molecules in solution, allowing transfollicular delivery of agents. This is an attractive route for transfollicular vaccinations as labile peptide and DNA-based vaccines can be delivered non-invasively to the perifollicular antigen presenting cells [9]

\section{Site of delivery}

A crucial factor that determines the strategies to be used for nanoparticle-based delivery through the skin is the desired site of action of the drug. Regional accumulation in the dermis is desired for the effect of many drugs that are intended for treatment of skin conditions. On the other hand, the concept of a systemic drug delivery by exploiting the large surface area and vascularity of the skin is far more challenging. Preliminary attempts have been made to deliver proteins and large macromolecules through the skin using nanoparticle and physical triggers. Insulin delivery is an attractive application of such technologies, and can make a tremendous impact if successful.

\section{Toxicity concerns}

The nature of the nanoparticle determines the cellular response when applied dermally. Enhanced permeation of certain non-degradable nanoparticles is undesired and leads to local and systemic toxicity. Titanium dioxide nanoparticles have been used as a component of sunscreens. The degree of aggregation of the titanium dioxide nanoparticles determines 
their effective size and hence penetration through the skin [10]. The type of nanoparticle and the skin condition will determine the penetration and toxicity. While a recent study provides laser scanning confocal microscopic evidence that polystyrene nanoparticles of 20 to $200 \mathrm{~nm}$ fail to penetrate through intact skin [11], others have shown a follicular penetration of the nanoparticles and corresponding toxic effects.

\section{Clinical implications}

Nanotechnology platforms may be designed to pass through the skin and be detectable systemically. This is desirable for a non-invasive drug delivery and can be used for labile molecules. However, the possibility of engineered nanoparticles penetrating through skin must be kept in mind when designing cosmetics and during evaluation of occupational exposure to other engineered nanoparticles.

Delivery of drugs through the skin can prove particularly attractive for drugs which have a low oral bioavailability and a high first-pass metabolism. Similarly, it would be very useful to deliver labile molecules like peptides, DNA through skin. The altered state of the skin can have a direct influence on nanoparticle penetration. For example, inflamed skin as seen in dermatitis can enhance the penetration of nanoparticles and cause preferential accumulation around inflamed hair follicles and sebaceous glands [12]. On the other hand, psoriatic plaques with epidermal thickening can reduce the penetration of drugs, requiring additional penetration enhancing strategies.

Vaccines are another attractive option as the skin is a highly immune responsive tissue having an abundance of antigen presenting cells like dendritic cells and Langerhans cells which can be accessed by transdermal delivery. Coreshell nanoparticles of PLGA and positively charged glycolchitosan have been used to demonstrate the feasibility of DNA delivery to Langerhans cells which later migrate to the draining lymph nodes suggesting a potential for exploitation of similar platforms for vaccine delivery [13].

Delivery of nutrients like vitamins, growth factors are another promising area where local delivery of the nutrients can help in maintaining the health of the skin and appendages and have found a role in cosmetics and anti-ageing products.

The size, composition, charge and the status of the skin (intact or injured) all play a role in determining the extent of penetration. Similarly, the degradability, surface chemistry and shape play important roles in determining the safety of the nanoparticles on systemic exposure through the skin. A clear understanding of these factors can help in the design of pharmacological agents being delivered efficiently through the skin while avoiding the toxic effects of undesired penetration.

\section{Translational challenges}

While the advent of nanotechnology has made the stratum corneum barrier more accessible, the translation of nanoparticle-based products for transdermal delivery will depend on their dose optimisation, precise control over their penetration and biodistribution. Many of the effects appear to be crucially dependent on size and charge; hence, it is critical to be able to reproducibly produce electrokinetically stable nanoparticles of narrow size distributions on scale up. At the same time, caution must be exercised over the accidental exposure and penetration of non-degradable nanoparticles that may cause toxicity concerns. Addressing these issues can lead to the development of many smart nanomaterial-based strategies in the future for exploiting transdermal delivery more efficiently.

Conflict of interest The author's research in transdermal delivery is funded by a grant from the Bill \& Melinda Gates Foundation through the Grand Challenges Exploration initiative and has a pending patent application in this area. The author has no other relevant affiliations or financial involvements with any organisation or entity with a financial interest or conflict of interest with the subject matter discussed in the manuscript.

\section{References}

1. Bolzinger MA, Briancon S, Pelletier J, Chevalier Y. Penetration of drugs through skin, a complex rate controlling membrane. Curr Opin Colloid Interface Sci. 2012;17:156-65.

2. Labouta HI, Schneider M. Interaction of inorganic nanoparticles with the skin barrier: current status and critical review. Nanomedicine: Nanotechnol Biol Med. 2013;9:39-54.

3. Charoenputttakhun P, Opanasopit P, Rojanarata T, Ngawhirunpat T. All trans-retinoic acid loaded lipid nanoparticles as a transdermal drug delivery carrier. Pharm. Dev. Technol. 2013; (in press).

4. Tomoda K, Terashima H, Suzuki K, Inagi T, Terada H, Makino K. Enhanced transdermal delivery of indomethacin using combination of PLGA nanoparticles and iontophoresis in vivo. Colloids Surf B Biointerfaces. 2012;92:50-4.

5. Lopez RF, Seto JE, Blankschtein D, Langer R. Enhancing the transdermal delivery of rigid nanoparticles using the simultaneous application of ultrasound and sodium lauryl sulphate. Biomaterials. 2011;32(3):933-41.

6. Ramadan S, Guo L, Li Y, Yan B, Lu W. Hollow copper sulphide nanoparticle mediated transdermal drug delivery. Small. 2012; $8(20): 3143-50$.

7. Desai P, Patlolla RR, Singh M. Interaction of nanoparticles and cell-penetrating peptides with skin for transdermal delivery. Mol Membr Biol. 2010;27(7):247-59.

8. Bachy V, Hervouet C, Becker PD, et al. Langerin negative dendritic cells promote potent $\mathrm{CD} 8^{+} \mathrm{T}$-cell priming by skin delivery of live adenovirus vaccine microneedle arrays. PNAS. 2013. doi: 10.1073/pnas. 1214449110.

9. Mittal A, Raber AS, Schaefer UF, et al. Non-invasive delivery of nanoparticles to hair follicles: a perspective for transcutaneous immunization. Vaccine. 2013. doi:10.1016/j.vaccine.2012.12.048. 
10. Bennett SW, Zhou D, Mielke R, Keller AA. Photoinduced disaggregation of $\mathrm{TiO} 2$ nanoparticles enables transdermal penetration. PLoS One. 2012;7(11):e48719. 1-7.

11. Campbell C, Contreras-Rojas LR, Delgado-Charro MB, Guy RH. Objective assessment of nanoparticle disposition in mammalian skin after topical exposure. J Control Release. 2012;162:201-7.
12. Abdel-Mottaleb MMA, Moulari B, Beduneau A, Pellequer Y, Lamprecht A. Nanoparticles enhance therapeutic outcome in inflamed skin therapy. Eur J Pharm Biopharm. 2012;82:151-7.

13. Lee PW, Hsu SH, Tsai JS, et al. Multifunctional core-shell polymeric nanoparticles for transdermal DNA delivery and epidermal Langerhans cells tracking. Biomaterials. 2010;31(8):2425-34. 